\title{
Interactive comment on "Evaluation of the
} University of Victoria Earth System Climate Model version 2.10 (UVic ESCM 2.10)" by Nadine Mengis et al.

\section{Anonymous Referee \#1}

Received and published: 15 March 2020

Review of Mengis et al. gmd-2019-373

This seems like a really straightforward behavior. The descriptions are excellent, and I'm quite impressed with the results of the model. I only have a few minor comments.

Abstract: Your abstract is pretty short and not all that specific. You have room to go into more details about some major developments or some details about how well the new version of the model performs.

Page 1, Lines 5-6: I think it would be helpful to be more specific here. More specifically, it is part of CMIP6, but the EMIC intercomparison - please say that. 
Page 4, line 33: Could you include a definition or short description of cryoturbation?

Page 5, line 16: Change citep to citet.

Page 6, line 7: Please use proper scientific notation - this is a bit confusing as written.

Page 6, line 15: Some grammar issues

Page 8, line 8: Can you go into a few more details as to why this might be problematic?

Page 8, lines 26-27: It might be outside the EMIC range but is well within the ESM range. Is that coincidental or because the code modifications have resulted in the ability to capture more complex behavior?

Interactive comment on Geosci. Model Dev. Discuss., https://doi.org/10.5194/gmd-2019-373, 2020. 